\title{
Perspectiva da exposição aos medicamentos na tentativa de suicídio
}

\author{
Perspective of exposure to drug in the suicide attempt \\ Perspectiva de exposición a drogas en el intento de suicidio
}

\section{Resumo}

O suicídio é considerado um problema grave de saúde pública, em média, 800 mil pessoas vão a óbito por suicídio no mundo por ano. O objetivo deste estudo foi avaliar o cenário da exposição a medicamentos associados à tentativa de suicídio. Trata-se de um estudo documental, retrospectivo, com abordagem quantitativa, realizado no período de janeiro a junho de 2020 em um centro de referência do estado do Ceará. Observaram-se 1.205 casos notificados de intoxicação aguda, sendo $69,2 \%$ destes por tentativa de suicídio. Destacaram-se os pacientes com faixa etária entre 20 e 29 anos, tanto do sexo feminino $(18,9 \%)$ quanto do sexo masculino $(13,2 \%)$. Com maior frequência relacionada a casos leves com 51,2\%. Os principais medicamentos envolvidos nas intoxicações foram ansiolíticos representando $18,6 \%$ dos casos e em $92,57 \%$ ocorreram nas residências dos pacientes. Conclui-se que a população precisa ser conscientizada dos riscos relacionados aos medicamentos, desde seu uso sem prescrições médicas até o abuso intencional.

Palavras-chave: Intoxicação; Medicamentos; Suicídio; Toxicologia.

\section{Abstract}

Suicide is considered a serious public health problem, on average 800 thousand people die from suicide in the world each year. The aim of this study was to assess the scenario of exposure to medications associated with a suicide attempt. This is a documentary, retrospective study with a quantitative approach, carried out from January to June 2020 in a reference center in the state of Ceará. There were 1,205 reported cases of acute intoxication, $69.2 \%$ of these 
due to attempted suicide. Patients aged between 20 and 29 years, both female (18.9\%) and male (13.2\%), stood out. More often related to mild cases with $51.2 \%$. The main drugs involved in intoxications were anxiolytics, representing $18.6 \%$ of the cases and in $92.57 \%$ they occurred in the patients' homes. It is concluded that the population needs to be made aware of the risks related to medications, from their use without medical prescriptions to intentional abuse.

Keywords: Intoxication; Medicines; Suicide; Toxicology.

\section{Resumen}

El suicidio se considera un grave problema de salud pública, en promedio 800 mil personas mueren por suicidio en el mundo cada año. El objetivo de este estudio fue evaluar el escenario de exposición a medicamentos asociados a un intento de suicidio. Se trata de un estudio documental, retrospectivo con enfoque cuantitativo, realizado de enero a junio de 2020 en un centro de referencia en el estado de Ceará. Se notificaron 1.205 casos de intoxicación aguda, el $69,2 \%$ de ellos por intento de suicidio. Destacaron los pacientes con edades comprendidas entre 20 y 29 años, tanto mujeres $(18,9 \%)$ como hombres $(13,2 \%)$. Más a menudo relacionado con casos leves con 51,2\%. Los principales fármacos implicados en las intoxicaciones fueron los ansiolíticos, representando el 18,6\% de los casos y en el 92,57\% ocurrieron en los domicilios de los pacientes. Se concluye que la población necesita ser consciente de los riesgos relacionados con los medicamentos, desde su uso sin prescripción médica hasta el abuso intencional.

Palabras clave: Intoxicación; Medicamentos; Suicidio; Toxicologia.

\section{Introdução}

De acordo com os dados da Organização Mundial da Saúde (OMS), a cada 40 segundos uma pessoa comete suicídio no mundo. Os métodos mais usados são intoxicação por medicamentos, enforcamento, arma de fogo e envenenamento por pesticidas. A taxa de suicídios, a cada 100 mil habitantes, aumentou cerca de 7\% no Brasil em 2016, ao contrário do índice mundial, que caiu 9,8\%. A incidência do suicídio é cerca de 80\% em países subdesenvolvidos (OMS, 2019).

Segundo a literatura, o suicídio é considerado um problema grave de saúde pública, em média, 800 mil pessoas ao ano vão a óbito por suicídio no mundo, principalmente os jovens entre 15 e 29 anos, que sofrem com problemas relacionados a autoaceitação, endividamento, doenças crônicas, conflitos familiares, desemprego e sexualidade (OPAS/OMS, 2016). Pode-se definir o suicídio como um ato intrépido, auto executado, com a intenção de pôr fim à própria vida, por meio de recursos percebidos como fatais. Trata-se de um comportamento influenciado por fatores de risco gerais e específicos, atrelados à complexa interação de aspectos psicológicos, biológicos, genéticos, culturais e/ou socioambientais (Carbogim et al., 2019).

O uso irracional dos medicamentos é um fator importante nas intoxicações, sendo as crianças e os idosos as principais vítimas em decorrência da falta de informações sobre as doses, forma farmacêutica, duração do tratamento e até mesmo a dispensação desses medicamentos adequadamente. As intoxicações por medicamentos ocorrem por diversos fatores, dentre os principais destacam-se: administração acidental ou erros de prescrição, tentativas de autoextermínio e abuso (principalmente entre adolescentes e adultos) (Gonçalves et al., 2017; Araújo et al., 2020).

O Sistema Nacional de Informações Tóxico-Farmacológicas (SINITOX), em 2020, registrou 20.637 casos por intoxicação medicamentosa no Brasil. É o segundo colocado entre os agentes tóxicos mais utilizados, caracterizando a segunda causa de morte, com 138 óbitos, dos quais 49 foram por tentativas de suicídio (Sinitox, 2020).

Diante do exposto e ciente da necessidade de aprofundar os conhecimentos sobre a exposição aos medicamentos na tentativa de suicídio, o presente trabalho teve como objetivo avaliar o cenário da exposição a medicamentos associados à tentativa de suicídio.

\section{Metodologia}

Trata-se de um estudo documental, retrospectivo e com abordagem quantitativa, realizado no período de janeiro a junho de 2020, em um Centro de Informação e Assistência Toxicológica (CIATOX) do estado do Ceará.

Incluíram-se todos os registros de tentativas de suicídio por medicamentos ocorridos no período de janeiro de 2016 a dezembro de 2020. Foram estudadas as seguintes variáveis: nome do medicamento, procedência do paciente (capital ou 
interior), sexo, idade, circunstância, via de exposição, tratamento sugerido, gravidade e desfecho do caso. Excluíram-se as fichas incompletas.

As informações foram coletadas por meio dos relatórios do Sistema Brasileiro de Dados de Intoxicação (DATATOX) da Associação Brasileira de Centros de Informação e Assistência Toxicológica (ABRACIT). A análise dos dados foi realizada por meio do programa Excel $^{\circledR}$ versão 15.26. As variáveis categóricas foram apresentadas como frequências absoluta (n) e relativa $(\%)$.

Este trabalho foi aprovado pelo Comitê de Ética em Pesquisa da Universidade de Fortaleza (UNIFOR) e do Hospital Instituto Dr. José Frota (IJF) sob parecer de número 3.550.331 e CAAE: 17010019.9.3001.5047.

\section{Resultados e Discussão}

No período do estudo, observaram-se 1.205 notificações de intoxicações agudas por medicamentos, das quais, 69,2\% ( $n=834)$ ocorreram por tentativa de suicídio, foco deste trabalho, seguidos de intoxicação acidental ( $\mathrm{n}=229 ; 19,0 \%)$ e abuso $(\mathrm{n}=50 ; 4,15 \%)$.

Dos casos de tentativa de suicídio por intoxicação medicamentosa $(n=834)$, destacaram-se os pacientes com faixa etária entre 20 e 29 anos, tanto do sexo feminino ( $n=158 ; 18,9 \%)$ quanto do sexo masculino ( $n=110 ; 13,2 \%)$, seguido de pacientes entre 15 e 19 anos do sexo feminino $(n=122 ; 14,6 \%)$ e de 30 a 39 anos do sexo masculino (n=58; 7,0\%) (Figura 1$)$.

Figura 1. Distribuição dos casos de tentativa de suicídio por intoxicação medicamentosa segundo sexo e faixa etária.

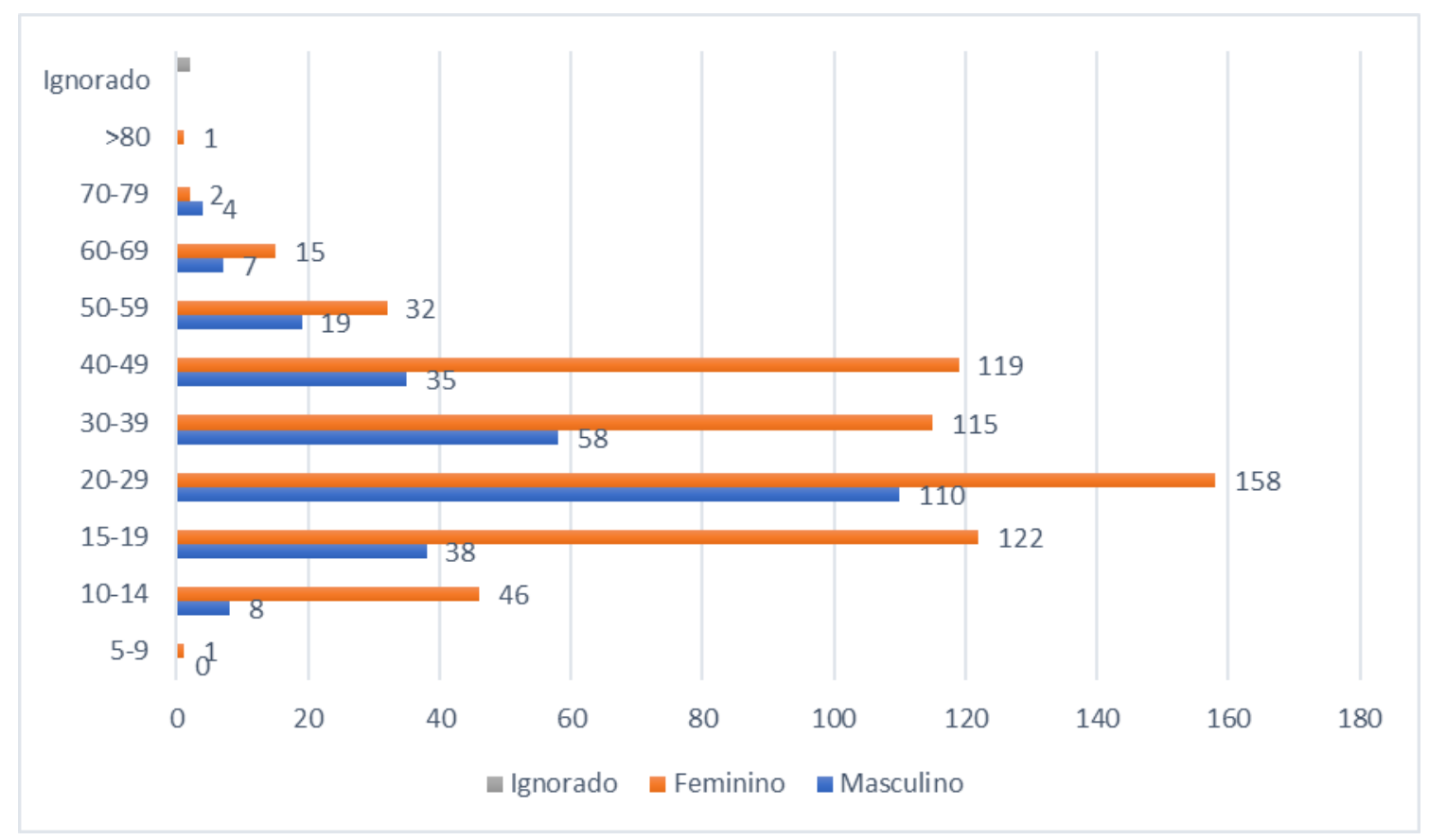

Fonte: Dados da pesquisa (2016-2020).

Quanto à ocupação, 22,9\% $(\mathrm{n}=190)$ dos pacientes eram estudantes e 10,3\% $(\mathrm{n}=86)$ profissionais do lar, enquanto 6,9\% (n=58) estavam desempregados no momento da ocorrência. Em 42,2\% (n=352) dos casos, a ocupação do paciente não foi informada. 
A Figura 2 apresenta a classificação da gravidade inicial das intoxicações. Houve prevalência de casos leves, com 51,2\% $(n=427)$ das ocorrências, seguido dos casos moderados $(n=223 ; 26,7 \%)$. Nos casos graves $(n=138 ; 16,5 \%)$, os pacientes apresentaram manifestações clínicas tais como: coma, depressão respiratória e pneumonia aspirativa.

Figura 2. Distribuição dos casos de tentativa de suicídio por intoxicação medicamentosa segundo a gravidade inicial.

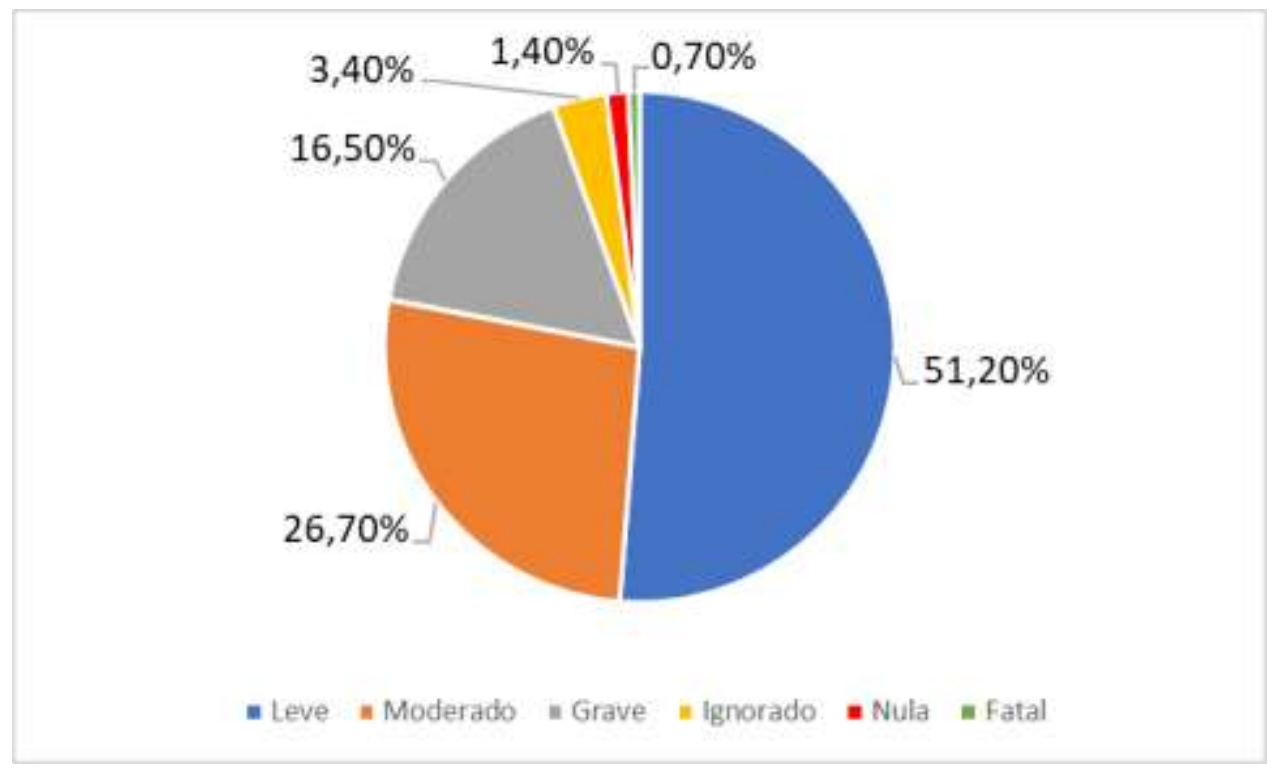

Fonte: Dados da pesquisa (2016-2020).

Na Tabela 1, observa-se que os sinais e sintomas das intoxicações ocorreram principalmente no sistema nervoso central (SNC) $(\mathrm{n}=926 ; 61,57 \%)$, seguido dos sintomas no trato gastrointestinal $(\mathrm{n}=211 ; 14,03 \%)$ e cardiovascular $(\mathrm{n}=113$; $7,51 \%)$.

Tabela 1. Distribuição dos casos de tentativa de suicídio por intoxicação medicamentosa segundo as manifestações clínicas dos pacientes.

\begin{tabular}{lcc}
\hline \multicolumn{1}{c}{ Sistemas acometidos } & \multicolumn{2}{c}{ Casos } \\
\cline { 2 - 3 } & N & \% \\
\hline Sistema Nervoso Central & 926 & 61,57 \\
Trato Gastrintestinal & 211 & 14,03 \\
Sistema Cardiovascular & 113 & 7,51 \\
Ignorado/Não informado & 96 & 6,38 \\
Aparelho respiratório & 58 & 3,85 \\
Órgãos dos sentidos & 58 & 3,85 \\
Músculo-Esquelético & 19 & 1,26 \\
Dermatológico & 14 & 0,93 \\
Genito-Urinário & 4 & 0,26 \\
Sistema Imunológico & 4 & 0,26 \\
Outros & 1 & 0,06 \\
\hline
\end{tabular}

Fonte: Dados da pesquisa (2016-2020). 
Os principais medicamentos envolvidos nas intoxicações foram os ansiolíticos representando 18,6\% ( $\mathrm{n}=286)$ dos casos, seguido dos antidepressivos com 18,0\% ( $\mathrm{n}=275)$ e antiepiléticos com 11,0\% (n=169) (Tabela 2).

Tabela 2. Distribuição dos casos de tentativa de suicídio por intoxicação medicamentosa segundo a classe farmacológica do medicamento.

\begin{tabular}{|c|c|c|c|c|c|c|c|}
\hline \multirow{2}{*}{$\begin{array}{c}\text { Classes de } \\
\text { Medicamentos }\end{array}$} & \multirow{2}{*}{$\begin{array}{l}\text { Cód. } \\
\text { ATC }\end{array}$} & \multicolumn{2}{|c|}{ Casos } & \multirow{2}{*}{$\begin{array}{c}\text { Classes de } \\
\text { Medicamentos }\end{array}$} & \multirow{2}{*}{$\begin{array}{l}\text { Cód. } \\
\text { ATC }\end{array}$} & \multicolumn{2}{|c|}{ Casos } \\
\hline & & $\mathbf{N}$ & $\%$ & & & $\mathbf{N}$ & $\%$ \\
\hline Analgésicos e & N02 & 129 & 8,39 & Cardiovasculares Anti & $\mathrm{C} 02$ & 97 & 6,31 \\
\hline Antipiréticos & & & & Hipertensivo & & & \\
\hline Ansiolíticos & N05B & 286 & 18,62 & Cardiovascular & $\mathrm{C} 10$ & 10 & 0,65 \\
\hline & & & & Hipolipemiantes & & & \\
\hline Antidepressivos & N06A & 275 & 18,0 & Cardiovasculares & $\mathrm{C} 01$ & 3 & 0,19 \\
\hline & & & & Terapia Cardíaca & & & \\
\hline Antiepiléticos & N03 & 169 & 11,0 & Cardiovasculares & $\mathrm{C} 05$ & 1 & 0,065 \\
\hline & & & & Vasoprotetor & & & \\
\hline Antipsicóticos & N05A & 149 & 9,7 & Descongestionantes & R01A & 6 & 0,39 \\
\hline Anestésicos & N01 & 1 & 0,065 & Digestivo & A09 & 2 & 0,13 \\
\hline Anti-helmínticos & $\mathrm{P} 02$ & 4 & 0,26 & Hipnóticos e Sedativos & N05C & 28 & 1,82 \\
\hline Anti-histamínicos & D04 & 68 & 4,43 & Hormônios sexuais & G03 & 6 & 0,39 \\
\hline Anti-inflamatório e & M01 & 46 & 3,0 & Laxantes & A07 & 1 & 0,065 \\
\hline Anti Reumático & & & & & & & \\
\hline Antianêmicos & B03 & 6 & 0,39 & Medicamento Não & - & 11 & 0,71 \\
\hline & & & & Determinado & & & \\
\hline Antibacterianos & J01 & 34 & 2,21 & $\begin{array}{l}\text { Medicamento de uso } \\
\text { tópico }\end{array}$ & D11 & 6 & 0,39 \\
\hline Antidiabéticos e & A10B & 14 & 0,91 & Medicamentos & V & 4 & 0,26 \\
\hline Insulinas & A10A & & & Fitoterápicos & & & \\
\hline Antieméticos e & A04 & 11 & 0,71 & Medicamentos & S01X & 1 & 0,065 \\
\hline Antinauseantes & & & & Oftálmicos & & & \\
\hline Antifúngicos & D01B & 1 & 0,065 & $\begin{array}{l}\text { Medicamentos para } \\
\text { doenças obstrutivas }\end{array}$ & R03D & 4 & 0,26 \\
\hline Antigotosos & M04 & 1 & 0,065 & $\begin{array}{l}\text { Medicamentos em } \\
\text { úlcera péptica }\end{array}$ & A02B & 18 & 1,17 \\
\hline Antimicobacterianos & J04 & 1 & 0,065 & $\begin{array}{l}\text { Medicamentos para } \\
\text { desordens do sistema }\end{array}$ & N07 & 14 & 0,9 \\
\hline & & & & nervoso & & & \\
\hline Antineoplásicos & L01 & 1 & 0,065 & $\begin{array}{l}\text { Outros produtos do } \\
\text { sistema digestório }\end{array}$ & A16 & 5 & 0,32 \\
\hline
\end{tabular}




\begin{tabular}{|c|c|c|c|c|c|c|c|}
\hline Antiobesidade & A08 & 1 & 0,065 & Preparações contra & $\mathrm{R} 05 \mathrm{~F}$ & 8 & 0,52 \\
\hline & & & & tosse e resfriado & & & \\
\hline \multirow[t]{2}{*}{ Antiparkinsoniano } & N04 & 9 & 0,58 & Psicoestimulantes e & N06B & 21 & 1,37 \\
\hline & & & & Nootrópicos & & & \\
\hline Antiprotozoário & $\mathrm{P} 01$ & 5 & 0,32 & Relaxantes musculares & M03 & 30 & 1,95 \\
\hline \multirow[t]{2}{*}{ Antitrombótico } & B01 & 1 & 0,065 & Substituto do sangue e & B05 & 1 & 0,065 \\
\hline & & & & soluções de perfusão & & & \\
\hline \multirow[t]{2}{*}{ Antivirais } & $\mathrm{J} 05$ & 3 & 0,19 & Terapêutica & H03 & 5 & 0,32 \\
\hline & & & & Tireoidiana & & & \\
\hline Antiácidos & $\mathrm{A} 02 \mathrm{~A}$ & 3 & 019 & Urológicos & G04 & 1 & 0,065 \\
\hline \multirow[t]{2}{*}{ Antídotos } & V03AB & 5 & 0,32 & Vitaminas e & A12 & 11 & 0,71 \\
\hline & & & & suplementos minerais & & & \\
\hline
\end{tabular}

Fonte: Dados da pesquisa (2016-2020).

Verificou-se que as tentativas de autoextermínio ocorrem na própria residência do paciente, com frequência de $92,6 \%$ $(\mathrm{n}=756)$. De acordo com o tempo entre a exposição e o atendimento, os pacientes procuraram e/ou foram encaminhados ao Centro de Referência em períodos de 24 minutos até 7 dias após o ocorrido, sendo que em 8,9\% (n=66) dos casos o atendimento foi realizado entre 2 a 3 horas, seguido do tempo de 4 a 5 horas com $8,7 \%(n=65)$.

Na Tabela 3, verifica-se que a conduta de desintoxicação com lavagem gástrica ocorreu em 16,5\% (n=371) dos casos e o uso de carvão ativado em dose única ou múltipla em $24,0 \%(n=541)$, seguido de tratamento sintomático e suportivo em $21,8 \%(n=490)$ das ocorrências.

Tabela 3. Distribuição dos casos de tentativa de suicídio por intoxicação medicamentosa segundo a conduta de tratamento sugerido.

\begin{tabular}{|c|c|c|c|c|c|}
\hline \multirow[t]{2}{*}{ Tratamento } & \multicolumn{2}{|c|}{ Casos } & \multirow[t]{2}{*}{ Tratamento } & \multicolumn{2}{|c|}{ Casos } \\
\hline & $\mathbf{N}$ & $\%$ & & $\mathbf{N}$ & $\%$ \\
\hline $\begin{array}{l}\text { Alcalinização sérica e } \\
\text { urinária }\end{array}$ & 31 & 1,37 & $\begin{array}{l}\text { Hidratação } \\
\text { venosa ou oral }\end{array}$ & 254 & 11,3 \\
\hline Antibioticoterapia & 5 & 0,22 & $\begin{array}{l}\text { Lavagem } \\
\text { gástrica }\end{array}$ & 371 & 16,5 \\
\hline Antídotos & 47 & 2,09 & Ignorado & 36 & 1,60 \\
\hline Aquecimento corporal & 1 & 0,04 & $\begin{array}{l}\text { Nenhum } \\
\text { tratamento }\end{array}$ & 4 & 0,17 \\
\hline Bicarbonato de Sódio & 32 & 1,42 & $\begin{array}{l}\text { Nutrição } \\
\text { parenteral }\end{array}$ & 1 & 0,04 \\
\hline Carvão ativado & 541 & 24,0 & $\begin{array}{l}\text { Observar sinais } \\
\text { e sintomas }\end{array}$ & 376 & 16,6 \\
\hline Corticoterapia & 3 & 0,13 & Outros & 6 & 0,26 \\
\hline Diazepam & 10 & 0,44 & Oxigênio & 3 & 0,13 \\
\hline Entubação endotraqueal & 5 & 0,22 & $\begin{array}{l}\text { Sintomático e } \\
\text { suportivo }\end{array}$ & 490 & 21,8 \\
\hline Hemodiálise & 22 & 0,98 & $\begin{array}{l}\text { Ventilação } \\
\text { mecânica }\end{array}$ & 9 & 0,40 \\
\hline
\end{tabular}

Antídotos: Atropina 9 (0,39\%); Biperideno 12 (0,52\%); Naloxona 2 (0,08\%); Flumazenil 22 (0,96\%); Vitamina K 2 $(0,08 \%)$. Fonte: Dados da pesquisa (2016-2020). 
Por fim, observou-se no desfecho, pacientes recebendo alta hospitalar sem manifestações clínicas (n=200 24,0\%) ou com manifestações clínicas leves $(n=353 ; 42,3 \%)$. Muitos casos com desfecho ignorado se deram pelo fato dos pacientes receberem alta hospitalar sem avaliação prévia do centro de intoxicação.

Figura 3. Distribuição dos casos de tentativa de suicídio por intoxicação medicamentosa segundo o desfecho.

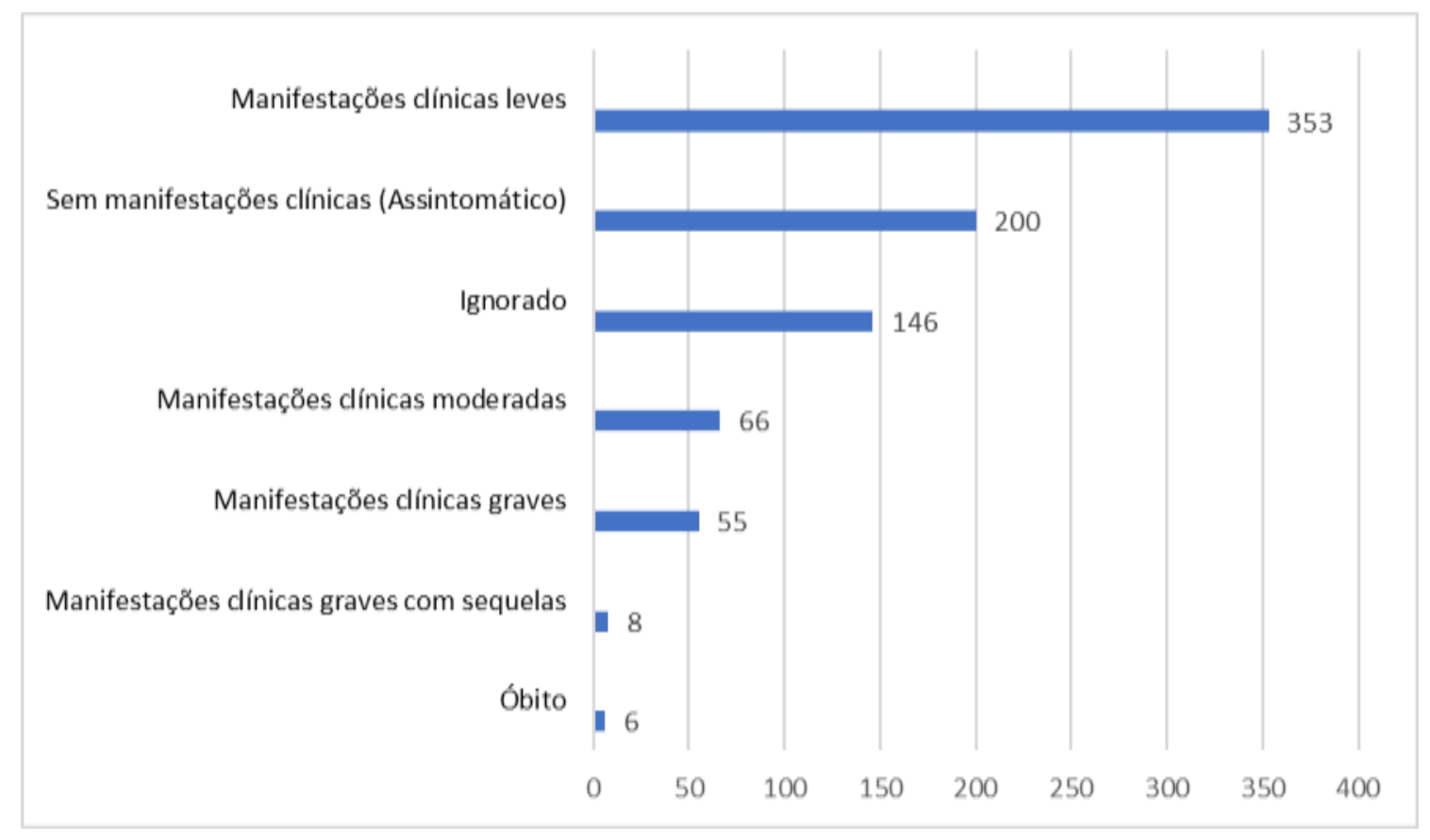

Fonte: Dados da pesquisa (2016-2020).

O predomínio do gênero feminino com faixa etária entre 20 e 29 anos e alto índice no número de intoxicação medicamentosa na tentativa de suicídio, está de acordo com o estudo de Chaves et al. (2017). Além disso, pessoas do sexo masculino que tentam suicídio por autointoxicação tendem a usar pesticidas ao invés de medicamentos (Bernardes; Turini; Matsuo; 2010). Ressalta-se também métodos radicais e violentos, tais como uso de armas de fogo ou enforcamento, são associados ao suicídio masculino (Baére; Zanello, 2020).

Normalmente, mulheres tentam o suicídio como fuga e esquecimento da vida presente, não como método de destruição e a literatura associa a tentativas de menor gravidade, principalmente devido ao uso de substâncias que apresentam baixa letalidade nas ocorrências. No entanto, vale salientar que as mulheres podem ser mais suscetíveis aos malefícios do uso de substâncias psicoativas devido a mudanças hormonais, estruturais e metabólicas (Trevisan; Santos; Oliveira, 2013).

Alguns marcos na vida da mulher adulta podem acarretar gatilhos para a tentativa de suicídio, como casamento, separação, gestação e problemas de saúde mental (Costa et al., 2021), além de fatores sociodemográficos diversos, que mudam segundo o contexto histórico, cultural e político (Felix et al., 2016). As frequências entre os homens da ocupação “desempregado" e entre as mulheres "donas-de-casa" condizem com a literatura (Bernardes; Turini; Matsuo, 2010).

Os dados demonstram uma baixa letalidade nas tentativas de suicídio envolvendo medicamentos. Ademais, a maior parte dos casos notificados teve desfecho final com manifestações leves. As principais classes farmacológicas encontradas nas referidas tentativas têm ação no SNC como os ansiolíticos, analgésicos e antidepressivos, e os sintomas mais frequentes foram: tonturas/vertigem, convulsões, sonolência/letargia e confusão mental, seguido dos sintomas gastrointestinais como vômitos, náuseas, diarreia, lesão esofágica e entre outros (Quan, 2016; Gussow; Carlson, 2018). 
Essas substâncias são comumente utilizadas nas autointoxicações, provavelmente devido à sua disponibilidade (analgésicos não opioides, por exemplo, possuem venda livre) e à maioria dos casos de suicídio envolverem algum transtorno mental ou sofrimento psíquico subjacente, cuja principal estratégia de tratamento nos diferentes países continua a ser farmacológica (Gussow; Carlson, 2018).

O uso do domicílio como local preferido para a tentativa de suicídio pode sugerir a desestruturação e desentendimentos familiares, situação frequente em lares de indivíduos que atentam contra a própria vida. Também como importante fator potencializador, a recorrência de tentativa de suicídio entre as mulheres pode ter retorno positivo em termos de pedido de socorro e/ou mudança de ambiente, o que encorajaria a repetição do ato. Outra situação que reflete uma prática comum à população brasileira é o estoque domiciliar de medicamentos. Seja resultante de automedicação, da prescrição em quantidades superiores às necessárias e/ou da não adesão ao tratamento. $\mathrm{O}$ acúmulo de medicamentos facilita o acesso e favorece a efetivação de um ato suicida por esse método (Trevisan; Santos; Oliveira, 2013; Tabosa et al., 2021).

Portanto, fica evidente a importância do farmacêutico no momento da dispensação do medicamento, incluindo orientações sobre o uso racional dos medicamentos, benefícios do tratamento, efeitos colaterais e males causados pelo uso incorreto dos medicamentos para que eventos de intoxicação sejam prevenidos (Malaman et al., 2009; Santana; Taveira; Eduardo, 2019).

Em relação ao tempo, os pacientes que procuram atendimento nas primeiras horas após a exposição são fundamentais para que possa ser feita uma desintoxicação adequada, com destaque, neste estudo, para lavagem gástrica e uso de carvão ativado em dose única e múltiplas. Para maior eficácia dessa abordagem inicial ao paciente intoxicado, o carvão ativado deve ser usado em até uma hora e a lavagem gástrica em até duas horas após a exposição (Albuquerque et al., 2017). Entretanto, foi analisado que alguns pacientes procuraram atendimento sete dias após a tentativa de suicídio.

Diante disso, é percebível que, mesmo com tantas dificuldades para fins de averiguação precisa da magnitude do suicídio no seio social, os resultados apontam que esse comportamento em regra, é praticado por adultos jovens e adolescentes, caracterizando importante problema de saúde pública, além de gerar consequências socioeconômicas negativas, visto que tais indivíduos ainda teriam muito a contribuir para o meio trabalhista e comunitário (Monte et al., 2016; Batista; Maranhão; Oliveira, 2018). A pesquisa envolvendo o tema deve ser constante, a fim de detectar fatores de risco, para que seja possível o desenvolvimento de estratégias para prevenção e assistência.

\section{Conclusão}

Há um alto índice de tentativas de suicídio por intoxicação medicamentosa, abrangendo adultos jovens, principalmente do sexo feminino. A população precisa ser conscientizada dos riscos dos medicamentos, desde seu uso sem prescrições médicas até o abuso intencional. Cabe aos serviços de prevenção e educação em saúde divulgar essas informações, notificar e educar seus usuários quanto a esta problemática. É importante lembrar que as ações sociais, familiares e profissionais devem se direcionar a favor da saúde mental dos indivíduos, proporcionando acolhimento, atenção e preocupação de modo a estimular esperança no indivíduo.

Faz-se necessário a realização de estudos em outros centros de informação e assistência toxicológica em diferentes regiões do País. No objetivo de investigarmos o cenário de intoxicações medicamentosas no território brasileiro.

\section{Referências}

Albuquerque, P. L. M. M. (Org). (2017). Intoxicações agudas: Guia prático para o tratamento. Fortaleza: Soneto Editora. https://saude.fortaleza.ce.gov.br/images/Manuais_saude/Guia_IJF_Intoxicacoes.pdf 
Research, Society and Development, v. 10, n. 11, e84101119273, 2021

(CC BY 4.0) | ISSN 2525-3409 | DOI: http://dx.doi.org/10.33448/rsd-v10i11.19273

Araújo, I. G. et al. (2020). Foco na automedicação em pacientes idosos. Brazilian Journal Of Development, 6(10), 81600-81607. https://www.brazilianjournals.com/index.php/BRJD/article/view/18805

Baére, F. \& Zanello, V. (2020). Suicídio e masculinidades: uma análise por meio do gênero e das sexualidades. Psicol. Estud, 25 , e44147. https://www.scielo.br/j/pe/a/LzMM7YDThptPXCkJkpKnWkn/?lang=pt

Batista, M. D., Maranhão, T. L. G., \& Oliveira, G. F. (2018). Suicídio em jovens e adolescentes: uma revisão acerca do comportamento suicida, sua principal causa e considerações sobre as formas de prevenção. Rev. Mult. Psic, 12(40), 705-719. https://idonline.emnuvens.com.br/id/article/view/1152/0.

Bernardes, S. S., Turini, C. A., \& Matsuo, T. (2010). Perfil das tentativas de suicídio por sobredose intencional de medicamentos atendidas por um Centro de Controle de Intoxicações do Paraná, Brasil. Cad. Saúde Pública, 26(7), 1366-1372. https://www.scielo.br/scielo.php?pid=S0102311X2010000700015\&script=sci_abstract\&tlng=pt.

Carbogim, F. C. et al. (2019). Suicídio e cuidado às vítimas de tentativa de suicídio. Revista de enfermagem, 13(4), 1090-1096. https://periodicos.ufpe.br/revistas/revistaenfermagem/article/view/238056

Chaves, L. H. S. et al. (2017). Intoxicação exógena por medicamentos: aspectos epidemiológicos dos casos notificados entre 2011 e 2015 no Maranhão. Revista Ciência \& Saberes - Facema, 3(2), 477-482. http://www.facema.edu.br/ojs/index.php/ReOnFacema/article/view/203

Costa, R. H. F. et al. (2021). Tentativas de suicídio associadas ao uso de medicamentos. Revista de Casos e Consultoria, 12(1), 23942. https://periodicos.ufrn.br/casoseconsultoria/article/view/23942.

Félix, T. A. et al. (2016). Fatores de risco para tentativa de suicídio: produção de conhecimento no Brasil. Revista Contexto \& Saúde, 16(31), 173-185. https://www.revistas.unijui.edu.br/index.php/contextoesaude/article/view/6079.

Gonçalves, C. A. et al. (2017). Intoxicação medicamentosa: relacionada ao uso indiscriminado de medicamentos. Revista Científica da Faculdade de Educação e Meio Ambiente, 8(1), 135-143. http://www.faema.edu.br/revistas/index.php/Revista-\%20FAEMA/article/view/449/442

Gussow, L. \& Carlson, A. (2018). Sedative hypnoptics in Rosen's Emergency Medicine.

Malaman, K. R. et al. (2009). Perfil das intoxicações medicamentosas, no Brasil. Infarma, 21(7/8), 9-15. http://revistas.cff.org.br/infarma/article/view/134

Monte, B. S. et al. (2016). Estudo Epidemiológico das intoxicações por medicamentos registradas pelo Centro de Informação Toxicológico do Piauí-CITOX. Revista Interdisciplinar, 9(3), 96-104. https://revistainterdisciplinar.uninovafapi.edu.br/index.php/revinter/article/view/481.

OMS. (2019). Setembro Amarelo: taxa de suicídio aumenta 7\% no Brasil em seis anos. https://pebmed.com.br/setembro-amarelo-taxa-de-suicidio-aumenta-7no-brasil-em-seis-anos/.

OPAS/OMS. (2016). Grave problema de saúde pública, suicídio é responsável por uma morte a cada 40 segundos no mundo. https://www.paho.org/pt/topicos/suicidio

Quan, D. (2016). Benzodiazepins in Tintinalli Emergency Medicine. https://accessmedicine.mhmedical.com/content.aspx?bookid=1658\&sectio nid $=109414024$

Santana, D. P. H., Taveira, J. C. F., \& Eduardo, A. M. L. N. (2019). A importância da atenção farmacêutica na prevenção de problemas de saúde. Revista de Iniciação Científica e Extensão (REICEN), 2(1), 59-60. https://revistasfacesa.senaaires.com.br/index.php/iniciacao-cientifica/article/view/235

Sinitox. (2020). Dados de agentes tóxicos. https://sinitox.icict.fiocruz.br/dados-de-agentes-toxicos.

Tabosa, Y. S. et al. (2021). Automedicação e riscos de intoxicação. Revista Científica Multidisciplinar Núcleo do Conhecimento, 9, 100-109. https://www.nucleodoconhecimento.com.br/saude/riscos-de-intoxicacao

Trevisan, E., Santos, J., \& Oliveira, M. (2013). Tentativa de suicídio de mulheres: Dados de um centro de assistência toxicológico do Paraná. Rev. Min Enferm., 17(2), 412-417. http://www.reme.org.br/artigo/detalhes/659. 